\title{
Manejo nutricional y de riego en minijardines clonales de Tectona grandis (Linn. F) en la zona sur de Costa Rica
}

\author{
Irrigation and nutritional management in clonal minigardens of Tectona grandis (Linn. F) \\ in the southern zone of Costa Rica
}

\author{
Fiorella Calderón-Ureña ${ }^{1}$ Edwin Esquivel-Segura² $\bullet$ Manuel Acevedo-Tapia ${ }^{3}$
}

\begin{abstract}
Forestry nutrition is a current development topic into in the nursery stages and also in plantations. In some cases there is evidence of nutrition deficiencies from the clonal minigardens, which are one of the initial stages in a clonal nursery. In a clonal minigarden with Tectona grandis we evaluated the irrigation system and treatment applications mixing fertilizer doses with varied irrigation criteria to apply the fertigation technique. The Christiansen and distribution uniformity coefficients were determined for the irrigation system. In addition to the treatments, the weekly factor was also used to evaluate the behavior of teak mother plants considering the sprouts harvest. The irrigation system showed results of its uniformity that placed it in an unacceptable level. Regarding the variable measures (diameter, height and number of sprouts) there were not significant differences $(p \leq 0.05)$ between treatments but if were between weeks. In this project we concluded that although there were not significant differences between treatments, the costs for using salts as fertilizer are considerably lower.
\end{abstract}

Key words: Nutritional deficiency, fertigation, teak, sprouts. 


\section{Resumen}

La nutrición forestal es un tema en desarrollo actual tanto en las etapas de vivero como en las plantaciones. En algunos casos se presentan evidencias de deficiencias nutricionales desde los minijardines clonales, los cuales son una de las etapas iniciales en un vivero clonal. En un minijardín clonal de Tectona grandis (Linn. F) se evaluó el sistema de riego y la aplicación de distintas dosis junto con criterios de riego variados para así poder aplicar la técnica de fertirriego. Se determinaron los coeficientes de uniformidad de Christiansen y de distribución. Aparte de los tratamientos, también se utilizó el factor de semanas medidas para evaluar el comportamiento de las plantas madre de la teca ante la cosecha de brotes. El sistema de riego fue catalogado como inaceptable por lo que no fue uniforme. En cuanto a las variables medidas (diámetro, altura y cantidad de brotes) no se encontraron diferencias significativas $(p \leq 0,05)$ entre tratamientos, pero sí entre semanas. En este proyecto se concluye que a pesar de que no existieron diferencias significativas entre tratamientos, los costos de utilizar sales como fertilizante son considerablemente menores..

Key words: Deficiencia nutricional, fertirriego, teca, brotes.

\section{Introducción}

Actualmente uno de los métodos más utilizados en el país para la reproducción de teca (Tectona grandis Linn.F.) son los viveros clonales mediante la técnica de propagación vegetativa. Esta alternativa trata de mejorar la producción, disminuir tiempos de enraizamiento, tener mejores respuestas a problemas fitosanitarios y mantener la producción de las plántulas durante todo el año a pesar de las condiciones desfavorables del tiempo [1].

Dentro de las etapas de los viveros clonales se encuentran los minijardines clonales. Aquí es donde están presentes las plantas madre, las cuales son escogidas de acuerdo a sus árboles parentales, quienes son el éxito del mejoramiento genético [2]. La función principal de las plantas madre es la producción de brotes.

Con el fin de mantener una producción de brotes constante es necesario mantener a las plantas madre dentro de un invernadero para que estén en un ambiente controlado. Entre las variables a tomar en cuenta se encuentran la temperatura, humedad, condiciones sanitarias y además un adecuado manejo de riego y nutrición [3], [4].
De acuerdo con McDonald [5] el conocer el momento adecuado para regar y la manera para realizar la actividad puede prevenir el daño por calor y disminuir el estrés hídrico de las plantas provocado por la humedad. También se debe de tener en cuenta que un mal diseño de riego puede producir aplicaciones de agua excesivas o insuficientes lo que provocaría baja efectividad y afectación directa en el desarrollo de las plantas en el minijardín.

Por otro lado, la fertilización es otra variable que influye directamente en la calidad de las plantas [6]. Las plantas responden diferente ante la aplicación de distintas dosis de fertilizante, por lo que es necesario realizar investigaciones acerca de la respuesta que tienen ante las dosis [7]. De acuerdo con Chávez y Fonseca [7] la aplicación de N, P y K favorecen el crecimiento, color y vigor de la teca en la etapa de viveros.

La empresa American Reforestation Company cuenta con minijardines clonales de teca. No obstante, las técnicas de nutrición que la empresa utiliza no están respaldadas con criterios de riego y fertilización definidos y fundamentados. Es por eso que se cree que con la optimización en el manejo nutricional y del riego del minijardín, se incrementará la producción y la calidad de brotes en las plantas madre.

El objetivo de este proyecto fue mejorar estado nutricional de los minijardines clonales de Tectona grandis (Linn F.) ubicados en la zona sur del país.

\section{Materiales y métodos}

El proyecto se estableció en el vivero clonal de la empresa American Reforestation Company ubicado en la Península de Osa, específicamente en Puerto Jiménez

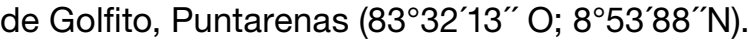

El sitio presenta clima tropical húmedo y muy lluvioso, con una precipitación que va desde los 2500 hasta los

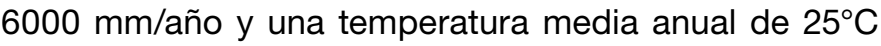
[8]. La estación seca está presente en los meses de diciembre a marzo, siendo marzo y febrero los meses más secos, mientras que la época lluviosa va desde abril hasta noviembre.

\section{Establecimiento de la línea base}

Se evaluaron 3 bancales cuyas mediciones promedio fueron de 21,05 m de largo y 1,36 de ancho. Se consultó a los encargados del vivero el horario, tiempo de duración y cantidad y método de aplicaciones tanto del riego como del fertilizante. También se obtuvo información 
relacionada al manejo y lavado de bancales, sustrato utilizado y periodo de renovación de plantas madre. Asimismo, se solicitó el precio de los productos utilizados.

\section{Propiedades físicas del sustrato de los bancales}

Se identificó el color de una muestra del sustrato tanto en húmedo como en seco con ayuda de la tabla Munsell. Por otro lado, se realizó una granulometría a la misma muestra con tamices de distintos tamaños $(8,6.7,2$ y 1 $\mathrm{mm}$ ). Luego de tamizado se pesó el sustrato que no pasó cada tamiz para determinar el porcentaje de cada uno.

\section{Homogeneidad del riego}

Se determinó el coeficiente de uniformidad de Christiansen [9]. (CUC) y coeficiente de uniformidad de distribución (CUD) para conocer la uniformidad del riego de los 3 bancales. Las fórmulas utilizadas se representan en las ecuaciones 1 y 2 .

$$
\begin{gathered}
C U C=\left[1-\frac{\sum_{i=1}^{n}\left|X_{i}-X_{m}\right|}{n * X_{m}}\right] * 100 \\
C U D=\frac{X_{25}}{X_{m}}
\end{gathered}
$$

Donde: $x_{i}$ es la cantidad de agua recogida por colector; $x_{m}$ es el valor medio de la cantidad de agua recogida, $x_{25}$ es la media de $25 \%$ del total de recipientes con la menor cantidad de agua recogida $(\mathrm{mm}) ; n$ es el número de recolectores.

Para colectar el agua del riego se utilizaron 63 recipientes de vidrio de $4,7 \mathrm{~cm}$ de diámetro por bancal y se enumeraron de forma consecutiva. En cada bancal se colocaron tablas de madera cada metro para un total de 21 tablas. Se ubicaron 3 recipientes en cada tabla manteniendo el orden con respecto a su enumeración, uno en el centro y 2 a los lados (figura 1). Se aplicó riego por un tiempo de 2,5 min, finalizado este periodo se determinó el volumen de agua recogida mediante el pesaje de los recipientes con una balanza electrónica $( \pm 0,1 \mathrm{~g})$.

\section{Densidad aparente del sustrato}

Para la estimación de la densidad aparente se utilizaron 8 cilindros de $5 \mathrm{~cm}$ de alto y $5 \mathrm{~cm}$ de diámetro, los cuales se enterraron entre los $5-10 \mathrm{~cm}$ de profundidad de cada bancal. Seguidamente se retiraron con el cuidado de que no se saliera el material y cada cilindro fue tapado por los 2 lados. Posteriormente se obtuvo el peso húmedo y el peso seco de cada muestra al secando estas por 24 horas a $105^{\circ} \mathrm{C}$. A cada cilindro se le determinó el volumen y se procedió a calcular la densidad aparente mediante la ecuación 3.
En cuanto al contenido de humedad se obtuvieron 15 muestras del sustrato del minijardín momentos antes del riego. Cada muestra se pesó para conocer su peso húmedo y luego se secó en horno por 24 horas a $105^{\circ} \mathrm{C}$ para el peso seco. Se determinó la retención de humedad con ayuda de la ecuación 4.

$$
\begin{gathered}
\frac{\text { Peso seco }(\mathrm{g})}{\text { Volumen }\left(\mathrm{cm}^{3}\right)} * 100 \\
\frac{\text { Peso húmedo }(\mathrm{g})-\text { Peso } \operatorname{seco}(\mathrm{g})}{\text { Peso } \operatorname{seco}(\mathrm{g})} * 100
\end{gathered}
$$

\section{Identificación de nutrientes}

Se obtuvo una muestra del agua utilizada para el riego de $250 \mathrm{ml}$, en este caso es agua de pozo. La muestra almacenada y enviada al laboratorio de la Universidad de Costa Rica para la realización de un análisis químico completo.

\section{Estrategia de mejoramiento}

Se realizó un ensayo en donde se utilizaron los métodos de la empresa y 2 dosis de fertilización y dos criterios de riego escogidos con ayuda de expertos nutricionales y con el análisis químico, en total existieron 9 tratamientos (cuadro 2). Para cada dosis nutricional se investigó el costo requerido.

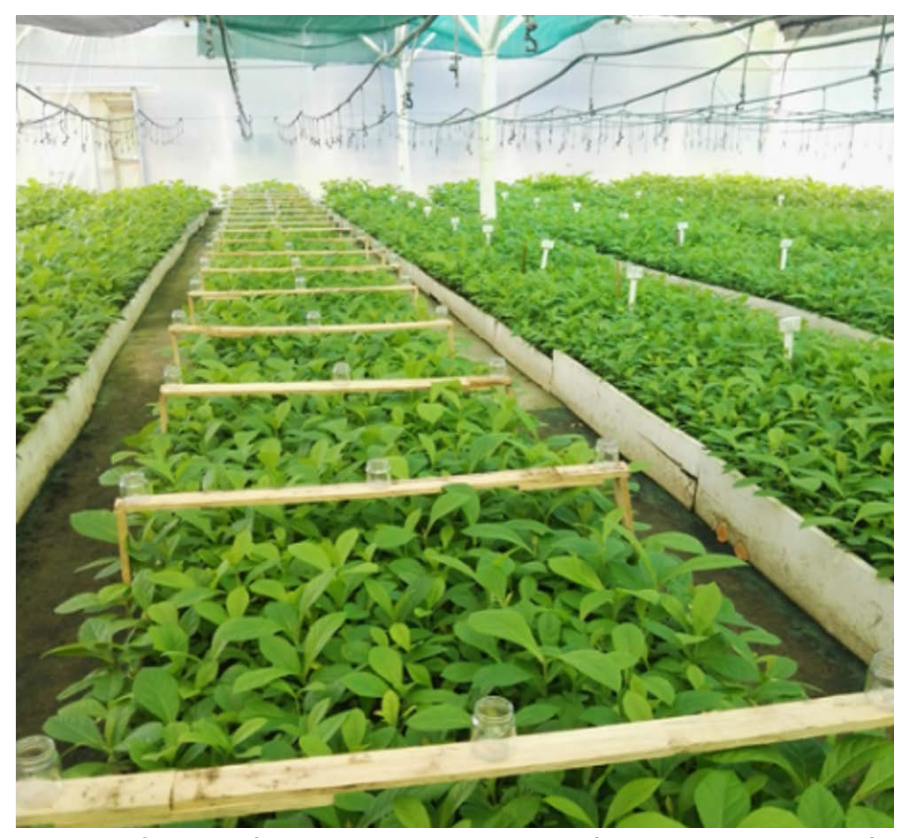

Figura 1. Colocación de frascos en el minijardín para la determinación del CUC y CUD, Puntarenas, Costa Rica.

Figure 1. Placing of flasks in the minigarden to determine the CUC and CUD,Puntarenas, Costa Rica. 
Cuadro 1. Parámetros de clasificación del desempeño de sistemas de riego por aspersión con base en el coeficiente de uniformidad de Christiansen (CUC) y de distribución (CUD). [10], [11]

Table 1. Parameters of classification of the performance of sprinkler irrigation system based on the Christiansen Uniformity coefficient (CUC) and the distribution coefficient (CUD).

\begin{tabular}{|c|c|c|c|c|c|}
\hline & Excelente & Bueno & Razonable & Malo & Inaceptable \\
\hline CUC (\%) & $>90$ & $80-90$ & $70-80$ & $60-70$ & $<60$ \\
\hline CUD (\%) & $>84$ & $68-84$ & $52-68$ & $36-52$ & $<36$ \\
\hline
\end{tabular}

Cuadro 2. Tratamientos utilizados en el ensayo en minijardín clonal de Tectona grandis, Puntarenas, Costa Rica.

Table 2. Treatments used in the trial in clonal minigarden of Tectona grandis, Puntarenas, Costa Rica.

\begin{tabular}{|c|c|c|}
\hline Tratamiento & Riego & Fertilización \\
\hline 1 & A & D \\
\hline 2 & A & E \\
\hline 3 & A & F \\
\hline 4 & B & D \\
\hline 5 & B & E \\
\hline 6 & B & D \\
\hline 7 & C & E \\
\hline 8 & C & F \\
\hline
\end{tabular}

Donde.

A y D: Dosis confidencial de la empresa. Todos los días elementos mayores (mañana), menores (tarde) e individuales 1 cada día.

B: Fertirriego de día por medio

C: Fertirriego/ Riego de manera alternada

E: $1,6 \mathrm{~g} \mathrm{~L}^{-1} \mathrm{MgSO}_{4} ; 0,942 \mathrm{~g} \mathrm{~L}^{-1} \mathrm{CaNO}_{3} ; 0,578 \mathrm{~g} \mathrm{~L}^{-1}$ Urea; 0,714 g L-1 $\mathrm{KH}_{2} \mathrm{PO}_{4}$

$\mathrm{F}: 0,454 \mathrm{~g} \mathrm{~L}^{-1} \mathrm{MgSO}_{4} ; 0,300 \mathrm{~g} \mathrm{~L}^{-1} \mathrm{CaNO}_{3} ; 0,556 \mathrm{~g} \mathrm{~L}^{-1}$ Urea; 0,348 g L-1 $\mathrm{KH}_{2} \mathrm{P}$

\section{Diseño experimental}

Para la aplicación de los tratamientos se utilizó el diseño experimental de parcelas divididas con el diseño experimental de bloques completos al azar, en donde el factor A estaba representado por los tratamientos (cuadro 3) mientras que el factor B eran las fechas en las que se realizaron mediciones ( 7 semanas). Cabe destacar que los bloques escogidos para el proyecto fueron los que presentaban una mezcla de clones, por lo que cada parcela utilizada presenta todos los clones de manera desordenada y sin poder identificar a cual pertenece.

Cada bloque estaba representado por bancales cuyas medidas están en el cuadro 1. En cada bloque se establecieron 9 parcelas con medidas de $1,5 \times 0,8 \mathrm{~m}$ y en cada una se marcaron 42 plantas madre para realizar las mediciones. Los tratamientos se asignaron de manera aleatoria para cada bancal. Las variables a medir fueron la elongación, diámetro, número de hojas y número de brotes. Las mediciones se realizaron cada 7 días por un periodo de 7 semanas.

\section{Análisis de datos}

Se analizaron los datos utilizando los programas de Office Excel 2013, Infostat 2008 y Past3. Se realizaron pruebas de normalidad y homogeneidad de varianzas, ANDEVA, así como pruebas para mostrar diferencias significativas entre los distintos factores y variables evaluadas. Para identificar las diferencias entre medias se utilizaron pruebas estadísticas tanto paramétricas como no paramétricas, las cuales fueron las pruebas de Tuckey $(0,05)$ y Friedman $(0,05)$ respectivamente. Por otro lado se utilizó el programa QGIS 2.18.3 para analizar los datos de la uniformidad del riego. 
Cuadro 3. Tamaño y porcentaje de partículas presentes en cada bancal en el minijardín clonal de Tectona grandis, Puntarenas, Costa Rica.

Table 3. Size and percentage of particles present in each bank in the clonal minigarden of Tectona grandis, Puntarenas, Costa Rica.

\begin{tabular}{|ccccccc|}
\hline \multirow{2}{*}{ Tamiz $(\mathrm{mm})$} & \multicolumn{2}{c}{ Bancal 6} & \multicolumn{3}{c|}{ Bancal 7} & \multicolumn{3}{c|}{ Bancal 8} \\
\cline { 2 - 7 } & Sustrato $(\mathrm{g})$ & $\%$ & Sustrato $(\mathrm{g})$ & $\%$ & Sustrato $(\mathrm{g})$ & $\%$ \\
\hline 8 & 4,37 & 0,37 & 11,70 & 1,05 & 14,53 & 1,43 \\
\hline 6,7 & 7,46 & 0,63 & 7,85 & 0,70 & 5,59 & 0,55 \\
\hline 2 & 254,20 & 21,56 & 297,48 & 26,71 & 283,12 & 27,91 \\
\hline 1 & 302,59 & 25,66 & 389,73 & 34,99 & 273,51 & 26,96 \\
\hline$<1$ & 610,43 & 51,77 & 406,94 & 36,54 & 437,60 & 43,14 \\
\hline
\end{tabular}

Cuadro 4. Coeficiente de uniformidad de Christiansen y coeficiente de uniformidad de distribución para cada bancal en el minijardín clonal de Tectona grandis, Puntarenas, Costa Rica.

Table 4. Christiansen uniformity coefficient and distribution uniformity coefficient for each bank in the clonal minigarden of Tectona grandis, Puntarenas, Costa Rica.

\begin{tabular}{|ccccccc|}
\hline No. Bancal & \multicolumn{3}{c}{ CUC (\%) } & \multicolumn{3}{c|}{ CUD (\%) } \\
\hline 6 & 56,05 & \pm & 0,90 & 35,98 & $\pm 0,18$ \\
\hline 7 & 48,42 & \pm & 0,67 & 30,58 & $\pm 0,12$ \\
\hline 8 & 48,22 & \pm & 0,80 & 25,55 & $\pm 0,14$ \\
\hline
\end{tabular}

\section{Resultados y discusión}

\section{Línea base}

El color del sustrato fue homogéneo en los bancales con un resultado de café grisáceo en seco y café muy oscuro en húmedo. En cuanto a la granulometría, de acuerdo a Yong y Warketin [12] se obtuvo un porcentaje alto de arena (0,2-2 $\mathrm{mm}$ ) con un promedio de $54,88 \%$ entre los 3 bancales (cuadro 3). Para este promedio no se incluyeron las partículas menores a $1 \mathrm{~mm}$ debido a que dentro de ellas se encuentran arenas, arcillas y limos los cuales no se lograron determinar por falta de instrumentos.

Dentro del tamiz de $6,7 \mathrm{~mm}$ se encuentra las que se consideran piedras machacadas, lisas y pequeñas (2$3,5 \mathrm{~mm}$ ) mejor conocida como grava. Acevedo [13] menciona que no deberían de existir dentro del bancal debido a que cambia mucho la porosidad del medio y podría provocar heterogeneidad en las plantas.

Por otro lado se considera que los bancales son heterogéneos en cuanto al sustrato debido a sus diferencias en los porcentajes, lo cual indica que la porosidad y la capacidad de retención de humedad podrían verse diferenciadas entre bancales.
Cuadro 5. Densidad aparente de cada bancal en el minijardín clonal de Tectona grandis, Puntarenas, Costa Rica.

Table 5. Apparent density of each bank in the clonal minigarden of Tectona grandis, Puntarenas, Costa Rica.

\begin{tabular}{|ccc|}
\hline Bancal & $\begin{array}{c}\text { Densidad } \\
\text { aparente }\left(\mathrm{g}^{*} \mathrm{Cm}^{-3}\right)\end{array}$ & CV \\
\hline 6 & $1,25 \pm 0,09$ & 7,32 \\
\hline 7 & $1,24 \pm 0,06$ & 4,52 \\
\hline 8 & $1,24 \pm 0,09$ & 7,30 \\
\hline
\end{tabular}

\section{Homogeneidad del riego}

Los datos resultantes mostraron que de acuerdo al CUC y CUD el riego presente en el invernadero está en condiciones inaceptables de acuerdo al cuadro 1 y 6 , ya que el CUC mostró datos menores al $60 \%$ y el CUD menores al $36 \%$.

Keller y Bliesner [14] comentan que para que el sistema por aspersión tenga buenos niveles de eficiencia se requiere un valor mínimo de CUC $\geq 80 \%$, lo cual en este caso no se cumple. De acuerdo con

Burt et al. [15] algunos factores que influyen en la heterogeneidad de la distribución del agua son las variaciones de presión, el diseño de los aspersores, tamaño de boquilla, espaciamiento entre cada una y las condiciones climáticas.

Algunas de las posibles causas observadas en el invernadero es que existen boquillas en condiciones desfavorables, la ubicación de los aspersores no es homogénea en cuanto a la altura que presentan y también hay lugares del bancal en donde el agua no es suficiente y por ende se secan rápido.

En la figura 2 se observa la distribución de donde se muestreó el agua aplicada en cada bancal, siendo las 

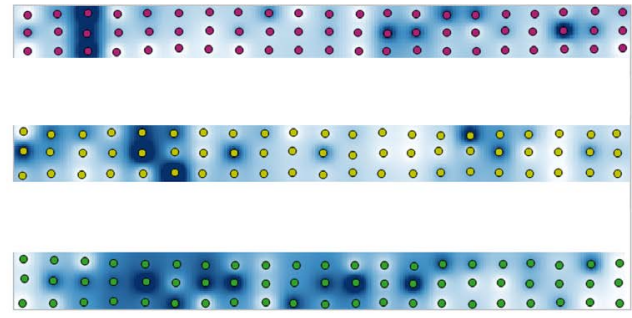

- Frascos bancal 8 - Frascos bancal 7 - Frascos bancal 6

Uuadro 2. Representación visual del agua de riego recolectada para cada bancal del minijardín clonal de Tectona grandis, Puntarenas, Costa Rica.

Figure 2. Visual representation of the irrigation water recollected for each bank in the clonal minigarden of Tectona grandis, Puntarenas, Costa Rica.

partes más oscuras donde se aplicó más agua y las partes más claras donde casi no se aplicó. Es evidente que en cada bancal existen zonas en donde la cantidad de agua es abundante comparada con otros sitios.

Paulino et al. [16] mencionan que el exceso de agua en ciertas partes del bancal provoca vulnerabilidad ante la existencia de lixiviación de nutrientes, reducción en la concentración de oxígeno disponible para las raíces y aumento en la incidencia de plagas y enfermedades. Por otro lado las zonas en donde el agua está escaseada, los riesgos de salinización del suelo incrementan e inhiben el potencial productivo.

Al comparar los promedios de los datos entre los bancales, los resultados mostraron que existen diferencia entre el bancal 6 y 8 con respecto al bancal 7 , siendo los promedios de 1.48, 0.96 y 1.29 para los bancales 6,7 y 8 respectivamente.

\section{Densidad aparente y contenido de humedad}

La densidad aparente resultó ser homogénea para los bancales evaluados (cuadro 5). Según Shargel y Delgado [17] los valores obtenidos corresponden a una textura fina arcillosa, ya que presentan una densidad aparente entre los 1,00-1,30 $\mathrm{g}^{*} \mathrm{~cm}^{-3}$. Donoso [18] comenta que valores bajos como los del proyecto indican que son sustratos que tienen buena porosidad, aireación, drenaje y buena penetración de raíces, por lo que es un resultado favorable para el crecimiento de las plantas madre.

En cuanto al contenido de humedad, de acuerdo al cuadro 6 las muestras presentaron diferencias significativas entre ellas al realizar una prueba Tuckey $(p \leq 0,05)$. Estos valores demuestran que no se tiene un criterio de riego estricto para los bancales, lo que podría suponer que no se esté regando en los momentos en que las plantas lo necesiten. Los resultados obtenidos podrían deberse al método que está utilizando la empresa actualmente,
Cuadro 6. Contenido de humedad de sustrato antes de ser regado en el minijardín clonal de Tectona grandis, Puntarenas, Costa Rica.

Table 6. Substrate moisture content before being irrigated in the clonal minigarden of Tectona grandis, Puntarenas, Costa Rica.

\begin{tabular}{|cc|}
\hline Muestra & Contenido de Humedad (\%) \\
\hline 1 & $17,37 \pm 2,19 \mathrm{a}$ \\
\hline 2 & $22,82 \pm 2,50 \mathrm{a}$ \\
\hline 3 & $14,09 \pm 3,95 \mathrm{~b}$ \\
\hline
\end{tabular}

el cual es de observación y tacto para conocer si el sustrato requiere ser regado.

\section{Identificación de nutrientes}

Con el análisis del agua de riego se propuso las fórmulas químicas (cuadro 2) de fertilización para aplicar a los bancales, esto debido a que los nutrientes que el agua les estaba proporcionando a las plantas a la hora de regar no estaba tomado en cuenta en la dosis utilizada por la empresa. Los resultados del análisis se muestran en el cuadro 7.

Para obtener las dosis nutricionales se tomó en cuenta principalmente los nutrientes de N, P y K. El porcentaje que está brindando el agua a las plantas madre es de $0,5 \%$ de Nitrógeno, $0,1 \%$ de fósforo y $4,1 \%$ de potasio. Sin embargo, Alvarado y Raigosa [20] mencionan que los valores óptimos para esta especie son de 1,52-2,78 $\mathrm{N}, 0,14-0,25 \mathrm{P}$ y $0,80-2,32 \mathrm{~K}$; aunque estos datos son considerados para plantaciones, es evidente que se deben hacer dosis nutricionales para poder suplir los requerimientos de las plantas madre.

\section{Estrategia de mejoramiento}

\section{Diámetro}

Esta variable no mostró diferencias significativas entre tratamientos pero sí entre semanas, tras haber variaciones tanto en aumento como en disminución de diámetro. En la figura 3 se puede observar que tratamientos como el T7, T8 y T9 tuvieron comportamientos más constantes al estar dirigidos mayormente hacia el aumento de la variable, esto teniendo en cuenta que estos 3 tratamientos se les aplicó la técnica de riego/fertirriego, por ende recibieron agua todos los días.

Los otros tratamientos si presentaron variaciones y algunas posibles causas pudieron ser por algún estrés hídrico que sufrieran las plantas madre al no ser regadas diariamente además del que ya sufren por la corta de brotes. Prieto et al. [21] comentan que el incremento en diámetro que sufre estrés hídrico es menor al de las que 
Cuadro 7.Análisis químico de agua utilizada para el riego en el minijardín clonal de Tectona grandis, Puntarenas, Costa Rica.

Table 7. Chemical analysis of the water used for the irrigation in the clonal minigarden of Tectona grandis, Puntarenas, Costa Rica.

\begin{tabular}{|c|c|c|c|c|c|c|c|c|c|c|}
\hline \multirow{2}{*}{$\mathrm{pH}$} & \multicolumn{9}{|c|}{$\mathrm{mg} / \mathrm{L}$} & \multirow{2}{*}{$\begin{array}{c}\mathrm{mS} / \mathrm{cm} \\
\mathrm{CE}\end{array}$} \\
\hline & $\mathrm{N}^{-} \mathrm{NH}^{4+}$ & $\mathrm{N}^{-} \mathrm{NO}^{3-}$ & N-UREICO & $\mathrm{Ca}$ & $\mathrm{Mg}$ & K & $P$ & $\mathrm{Na}$ & S & \\
\hline 7,3 & ND & 0,5 & ND & 28,6 & 15,6 & 4,1 & 0,1 & 6,2 & 0,8 & 0,3 \\
\hline
\end{tabular}

[19] *Fe, Zn, Cu y Mg no fueron identificados en la muestra.

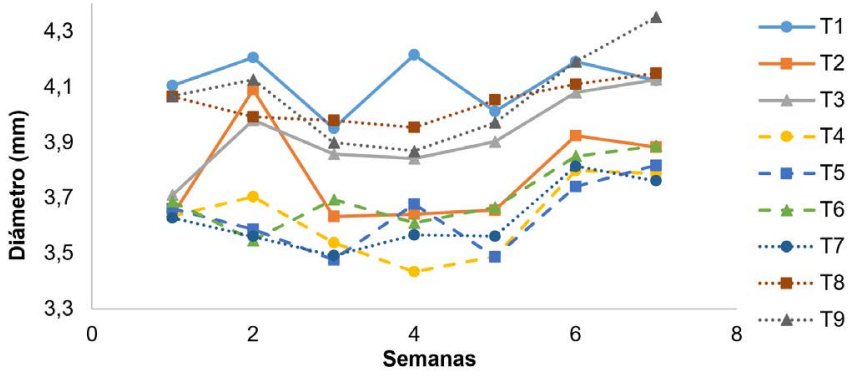

Figura 4.Altura promedio de plantas madre de Tectona grandis de acuerdo a la semana de medición en el minijardín clonal, Puntarenas, Costa Rica.

Figure 4. Average hight of mother plants of Tectona grandis according to me measure week in the clonal minigarden, Puntarenas, Costa Rica.

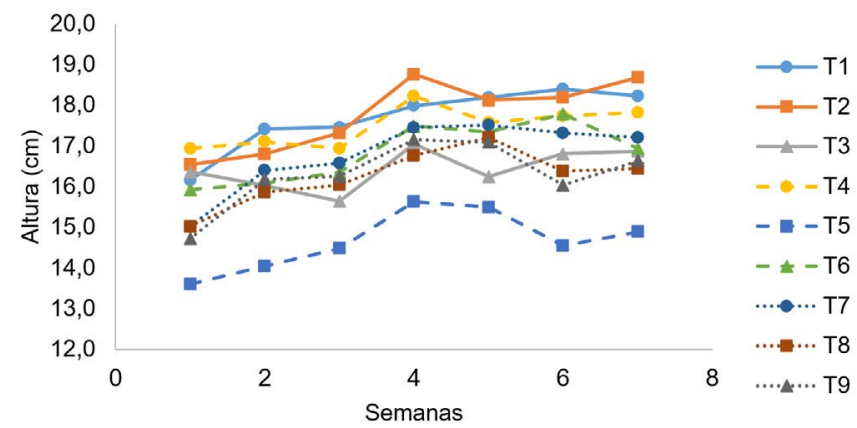

Figura 5. Cantidad de brotes promedio por planta madre de Tectona grandis de acuerdo a la semana de medición en el minijardín clonal, Puntarenas, Costa Rica.

Figure 5. Number of average shoots per mother plants of Tectona grandis according to the measure week in the clonal minigarden, Puntarenas, Costa Rica.

no sufren. Por otro lado, Landis et al. [22] mencionan que un sustrato con humedad alta previene la afectación por escasez hídrica al tener agua disponible fácilmente.

\section{Altura}

La variable altura no presentó diferencias significativas entre tratamientos. Sin embargo, en otros estudios de Pinus radiata la elongación si se diferenció al aumentar dosis de fertilizante, en este caso de sales nitrogenadas [23]. Igualmente sucedió en estudios con Eucalyptus globulus [24].

Por otro lado, sí existieron diferencias significativas entre semanas (figura 4), tanto en aumento como en disminución producto de la corta de brotes (semana 1, 2,5 y 6). Sin embargo, en las 2 primeras semanas no se logró ver cambios visibles debido a que previo a esas semanas pasó un periodo sin cosechar por lo que las plantas madre tomaron mucha altura y estas semanas funcionaron para nivelar el minijardín.

Es importante recalcar que para los tratamientos $1,4 \mathrm{y}$ 7 la dosis de fertilización no se aplicó completamente a partir de la semana 4 debido a un desabastecimiento de producto en la empresa. No obstante, esta situación no generó grandes diferencias en los resultados al presentar comportamientos similares a los demás.

\section{Brotes}

Para los tratamientos no se obtuvieron diferencias significativas al todos tener un promedio de 1,2-1,4 de brotes por planta madre. Un estudio de Chacón y Murillo [1] en minijadines de Gmelina arborea muestra que en promedio se cosechan 9,88 brotes por planta madre cada 15 días con un sustrato de tierra/arena, sin embargo, el distanciamiento de las plantas madre en el minijardín era de $40 \times 40 \mathrm{~cm}$ mientras que el del proyecto eran de $5 \times 5 \mathrm{~cm}$, provocando que se esté generando mayor competencia en alcanzar más altura para 1-2 brotes que en la producción de ellos.

En cuanto a las semanas por medio de una prueba Friedman $(p \leq 0,05)$ se presentaron diferencias significativas En la figura 5 se puede notar que la mayoría de los tratamientos a lo largo de la semana siguen la misma dinámica de aumentar brotes en las semanas 4 y 7 y disminuyen la cantidad en las semanas 2 y 5 producto de la cosecha. A parte de esto, de la semana 2 a la 3 los valores de brotes se mantienen constantes y de la 5 a la 6 varían tanto en aumento como en disminución debido a que en esa semana se cosechó en menor cantidad.

Además, se observa que a partir de la semana 6 los comportamientos de los tratamientos comienzan a variar 


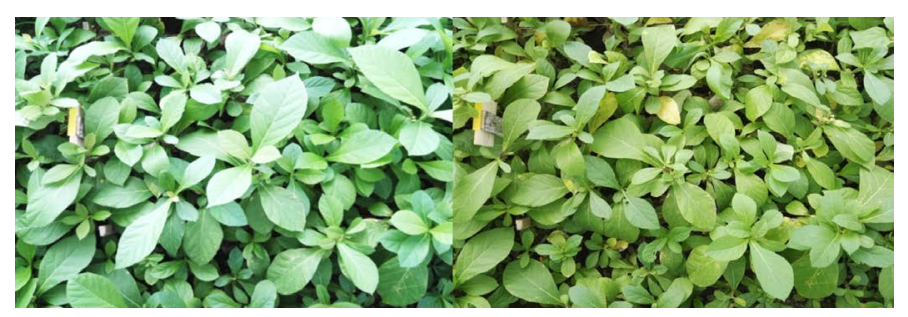

Figura 6. Visualización de cantidad de hojas en plantas madre de Tectona grandis al comparar dos semanas seguidas de medición en el minijardín clonal, Puntarenas, Costa Rica.

Figure 6. Visualization of the number of leaves in mother plants of Tectona grandis when comparing two consecutive weeks of measurement in the clonal minigarden, Puntarenas, Costa Rica.

y una posible causa de ello es que la respuesta a los tratamientos se esté presentando a partir de la semana 6 en adelante. Esto porque previo al inicio de la aplicación de tratamientos no se lavaron los nutrientes presentes en los bancales debido a que, al estar el sustrato en contacto con el suelo, no se aseguraba que el lavado fuera efectivo y aparte las instalaciones no se prestaban para realizarlo.

Abdelnour, Valverde y Aguilar [25] en un ensayo que realizaron mostraron que utilizando distintos reguladores de crecimiento obtuvieron de 1 a 4,6 brotes por estaca de la Tectona grandis en un periodo de 6 semanas, lo cual se asemeja a los valores obtenidos en el proyecto, a pesar de que en este caso no se utilizó reguladores de crecimiento y lo que se evaluó fue plantas madre en vez de estacas.
Desde otro punto de vista se debe destacar que a pesar que las plantas madre pasaron por 4 cosechas durante el proyecto, cada semana se lograron obtener resultados positivos para una posible cosecha con excepción de la semana 5 y 7 con los tratamientos 5 y 6 respectivamente, los cuales obtuvieron un promedio inferior a 1 para la cantidad de brotes (figura 7). Además, las plantas madre incrementan el número de brotes conforme se producen podas o se cosechan los brotes [26] por lo que, si se hubiera extendido el periodo de medición, las plantas madre pudieran producir una mayor cantidad de brotes.

Para visualizar los cambios de una semana a otra se muestra la figura 6 , en donde las fotografías fueron tomadas en la semana 4 (izquierda) y en la semana 5 (derecha) y se puede observar que la densidad entre las imágenes es notoriamente distinta, ya que la cantidad de hojas grandes se redujo producto de la corta de brotes, lo que también llegó a disminuir la altura de las plantas madre.

\section{Costos}

Para realizar el cálculo de los costos solo se tomó como base el minijardín clonal de la empresa para no usar todo el vivero. De acuerdo a información obtenida de los precios y a los datos brindados por la empresa se logró calcular la cantidad de veces que se podría fertilizar en las diferentes dosis utilizadas en los bancales en relación al cuadro 2, los resultados se muestran a continuación (cuadro 8).

Con los cálculos mostrados se demuestra que, con el uso de sales, los fertilizantes pueden ser utilizados en un periodo de 2 a 3 años sin que se acabe el producto,

Cuadro 8. Rendimiento de las diferentes dosis utilizadas en el minijardín clonal de Tectona grandis, Puntarenas, Costa Rica.

Table 8. Performance of the diferent doses used in the clonal minigarden of Tectona grandis, Puntarenas, Costa Rica.

\begin{tabular}{|c|c|c|c|c|c|c|}
\hline & Químico & \multicolumn{2}{|c|}{ Dosis $40 \mathrm{lt}$} & Días & Mes & Año \\
\hline & Elementos mayores orgánicos & 200 & $\mathrm{ml}$ & 18,93 & 0,63 & \\
\hline & Elementos menores orgánicos & 200 & $\mathrm{ml}$ & 18,93 & 0,63 & \\
\hline & Elementos individuales orgánicos & 200 & $\mathrm{ml}$ & 18,93 & 0,63 & \\
\hline \multirow{4}{*}{ Dosis 1} & $\mathrm{MgSO}_{4}$ & 32 & $g$ & 781,25 & 26,04 & \multirow{4}{*}{2,17} \\
\hline & UREA & 11,6 & $g$ & 3879,31 & 129,31 & \\
\hline & $\mathrm{KH}_{2} \mathrm{PO}_{4}$ & 14,2 & $g$ & 1760,56 & 58,69 & \\
\hline & $\mathrm{CaNO}_{3}$ & 18,8 & g & 1329,79 & 44,33 & \\
\hline \multirow{4}{*}{ Dosis 2} & $\mathrm{MgSO}_{4}$ & 18,16 & $g$ & 1376,65 & 45,89 & \multirow{4}{*}{3,12} \\
\hline & UREA & 13,92 & $g$ & 3232,76 & 107,76 & \\
\hline & $\mathrm{KH}_{2} \mathrm{PO}_{4}$ & 22,24 & $g$ & 1124,10 & 37,47 & \\
\hline & $\mathrm{CaNO}_{3}$ & 12 & $g$ & 2083,33 & 69,44 & \\
\hline
\end{tabular}


mientras que los químicos de la empresa rinden para menos de 1 mes cada envase.

Cabe agregar que de acuerdo con Burt [27] el fertirriego es el único método correcto de aplicar fertilizantes a los cultivos bajo riego siendo este aplicado uniformemente. Una buena aplicación de fertirriego trae consigo reducciones en la aplicación de fertilizantes [28] y por ende menos costos. Imas [29] también menciona que el fertirriego es totalmente necesario cuando el método de riego es por presión, como los aspersores que la empresa utiliza.

Si el riego y la fertilización se realizan de forma separada utilizando sales, los beneficios del riego no se verán reflejados en el cultivo ya que es probable que haya zonas donde las sales no se logren disolver por falta de agua. Es por eso que incorporar los nutrientes dentro del sistema de riego es la mejor opción para que la fertilización se realice de manera uniforme.

Es evidente entonces que la empresa se vería beneficiada al practicar fertirriego con sales que con los compuestos orgánicos viéndolo tanto a nivel económico como nutricionalmente ya que se le estarían brindando los nutrientes exactos que la planta necesita y no una dosis que está generalizada.

\section{Conclusión}

La densidad aparente de los bancales permite que el sustrato presente buena aireación, buen drenaje y buenas condiciones para la penetración de raíces. El riego no es uniforme de acuerdo al CUC y CUD y no existe un criterio de riego definido.

Los análisis químicos mostraron que el sustrato se encuentra balanceado y las plantas presentan dosis de nutrientes mayores a las recomendadas.

A pesar que por un problema de abastecimiento no se logró tener la aplicación del tratamiento durante todo el periodo, durante las semanas de medición no se encontraron diferencias entre tratamientos.

Es posible que dosis de fertilización aplicada a los bancales en los distintos tratamientos mostraran efecto residual por influencia de los nutrientes brindados por la empresa en semanas anteriores.

Los costos de utilizar las sales son más económicos que los fertilizantes orgánicos.

\section{Recomendaciones}

Mejorar el sistema de riego de la empresa, el cual puede empezar por el mantenimiento de las boquillas y las mangueras utilizadas hasta lograr obtener valores iguales o superiores a $80 \%$ de CUC.

En un futuro ensayo de fertilidad se recomienda lavar el sustrato para que los nutrientes brindados previamente no influyan en los resultados obtenidos.

Cambiar el sustrato de los bancales a solamente arena para poder controlar de mejor manera la nutrición al ser sustrato inerte.

Las dosis de sales utilizadas pueden mejorarse al interpretar análisis foliares. Además, si se decidiera fertilizar utilizando sales, es recomendable añadir sulfatos de micronutrientes ( $\mathrm{Fe}, \mathrm{Mn}, \mathrm{Cu}, \mathrm{Zn}$, etc.).

Analizar la senescencia de las hojas para determinar cuánto tiempo duran las hojas vivas en la planta.

\section{Referencias}

[1] P. Chacón y O. Murillo, "Análisis comparativo de la producción de minijardines clonales hidropónicos y jardines clonales en tierra de melina (Gmelina arborea Roxb.)," Kurú: Revista Forestal, vol. 2, no. 6, pp. 2-5, 2005.

[2] C. M. Espitia, G. O. Murillo y P. C. Castillo, "Ganancia Genética Esperada en Teca (Tectona grandis L.f.) en Córdoba (Colombia)", Colombia Forestal, vol. 14, no. 1, pp. 83, 2011.

[3] M. E. Daorden, Cultivo in vitro de tejidos vegetales [Diapositivas de Power Point], 2007. Recuperado de https://inta.gob.ar/sites/default/files/script-tmpmd_0701.pdf. [Accesado: 5 de Octubre].

[4] A. N. Ayala-Terán, "Establecimiento de cultivo in vitro de Molle (Schinus molle L.) a partir de yemas axilares tomadas de plantas madre como una herramienta para la propagación de la especie en el distrito metropolitano de Quito", Tesis de licenciatura, Escuela Politécnica del Ejército, Sangolquí. 2011

[5] S. McDonald, Irrigation in Forest-Tree Nurseries: Monitoring and Effects on Seedling Growth, en Forestry Nursery Manual: Production of Bareroot Seedlings, M. L. Duryea, C. R. Perry y T. D. Landis. Dordrecht, Springer,1987, 141-159.

[6] N. Escamilla-Hernández, J. J. Obrador-Olán, E. CarilloÁvila y D. Palma-López, "Uso de fertilizantes de liberación controlada en plantas de teca (Tectona grandis), en la etapa de vivero", Revista Fitotecnia Mexicana, vol. 38, no. 3, pp. 329-333, 2015.

[7] E. Chaves, y W. Fonseca, Teca (Tectona grandis), especie de árbol de uso múltiple en América Central, 1991. 
Recuperado de http://cort.as/-KCl- [Accesado: 5 de Octubre].

[8] Oficina Subregional Osa y S. Amador, Caracterización del territorio Península de Osa. Instituto de Desarrollo Rural, 2016. Disponible en: https://www.inder.go.cr/territorios_ inder/region_brunca/caracterizaciones/Caracterizacionterritorio-Peninsula-Osa.pdf. [Accesado: 6 de Octubre].

[9] J. Christiansen, Irrigation by Sprinkling. Berkeley, California: Universidad de California, 1942. Recuperado de https://archive.org/stream/irrigationbyspri670chri\#page/ n0/mode/2up. [Accesado: 5 de Octubre].

[10] N. Flórez-Tula, I. Zution-Gonçalves, D. RodriguesCalvacante Feitosa, A. Agnellos-Barbosa, F. Ponciano-de Deus, M. Diego-Ribeiro y E. Eiji-Matsura, "Eficiencia de aplicación de agua en la superficie y en el perfil del suelo en un sistema de riego por aspersión", Agrociencia, vol. 47, no. 2, pp. 113, 2013.

[11] A. Bernardo, C. Alves-Soares y Chartuni-Mantovani, Manual de Irrigação. (Vol 8). Impressora Universitária. Viçosa/MG, 2009.

[12] R. N. Yong y B. P. Warkentin, Introduction to soil behavior. New York: MacMillan Co., 1966.

[13] M. Acevedo-Tapia. "Uso de sustratos en viveros" Comunicación personal mediante correo electrónico. 2018. 1 p.

[14] J. Keller y R. D. Bliesner, Sprinkle and Trickle Irrigation. New York: USA: Van Nostrand Reinhold, 1990.

[15] C. M. Burt, A. J. Clemmens, T. S. Srelkoff, K. H. Solomon, R. D. Bliesner, L.A. Hardy, T.A. Howell y D. E. Eisenhauer, "Irrigation Performance Measures: Efficiency and Uniformity", Journal of Irrigation and Drainage Engineering, vol. 123, no. 6, pp. 423-442, 1997.

[16] M. de O. Paulino, F. P. Figueiredo, R. C. Fernandes, J. L. Maia, D. O. Guilherme y F. S. Barbosa, "Avaliação da uniformidade e eficiência de aplicação de água em sistemas de irrigação por aspersão convencional", Revista Brasileira de Agricultura Irrigada, vol. 3, no. 2, pp. 48-54, 2009.

[17] W. R. Schargel y F. Delgado, Características y manejo de los suelos utilizados en la producción de carne en Venezuela, en VI Cursillo sobre Bovinos de Carne, D. Plasse, N. Peña de Borsotti. Maracay, FCV-UCV. 1990, 187-220.

[18] C. Donoso. Ecología forestal. Valdivia, Chile: Universidad Austral de Chile, 1992.

[19] CIA (Centro de Investigaciones Agronómicas), Reporte de ensayos. Ciudad de la investigación, Universidad de Costa Rica, 2018.

[20] A. Alvarado y J. Raigosa, Nutrición y Fertilización Forestal en Regiones Tropicales. San José, Costa Rica: Asociación Costarricense de la Ciencia del Suelo, 2012.

[21] J. A. Prieto-Ruiz, E. H. Cornejo-Oviedo, P. A. DomínguezCalleros, J. de J. Návar-Chaidez, J. G. MarmolejoMoncivais, y J. Jiménez-Pérez, "Estrés hídrico en Pinus engelmannii Carr., producido en vivero", Investigación Agraria: Sistemas de Recursos Forestales, vol.13, no. 3, pp. 443-451, 2004.
[22] T. Landis, R. Tinus, S. McDonald y J. Barnett, Seedling nutrition and irrigation. Washington, DC: U.S. Department. of Agriculture, Forest Service, 1989.

[23] C. A. Hernández, y P. R. Rubilar, "Efecto de la fertilización nitrogenada y fosforada en el desarrollo y fenología de brotes de setos de Pinus radiata", Bosque (Valdivia), vol. 33, no. 1, pp. 53-54, 2012.

[24] J. Monsalve, R. Escobar, M. Acevedo, M. Sánchez y R. Coopman, "Efecto de la concentración de nitrógeno sobre atributos morfológicos, potencial de crecimiento radical y estatus nutricional en plantas de Eucalyptus globulus producidas a raíz cubierta”,. Bosque, vol. 30, no. 2, pp. 88-94, 2009.

[25] A. Abdelnour, L. Valverde-Cerdas y M. E. Aguilar, Micropropagación de tres especies maderables de importancia económica y ecológica para Costa Rica. Proyecto de investigación interinstitucional. ITCRUNA-CATIE, 2004. Recuperado de http://cort.as/-KCl8. [Accesado: 25 de Octubre].

[26] A. Aparicio-Rentería, H. Cruz-Jiménez, y O. MontielRuíz, "Multiplicación clonal de pinos a través del uso de estacas: una alternativa para mantener ganancias genéticas forestales", Foresta Veracruzana, vol. 10, no. 1, pp. 54, 2008.

[27] C. M. Burt, "Fertigation Basics". Irrigation Training and Research Center. 1998. [En línea]. Disponible en: http:// www.itrc.org/papers/pdf/fertbasics.pdf

[28] C. M. Burt, "Fertigation - The Next Frontier," Irrigation Business and Technology, vol 4, no. 3, pp. 16-19, 1995.

[29] P. Imas, "Manejo de nutrientes por fertirriego en sistemas frutihorticolas". International Potash Institude, 1999. Recuperado de https://www.ipipotash.org/ar/presentn/ mdnpfesf.php?l=3. [Accesado: 25 de Octubre].

\section{Este artículo debe citarse como:}

Calderón-Ureña, F; Esquivel-Segura, E; Acevedo-Tapia, M. (2019). Manejo nutricional y de riego en minijardines clonales de Tectona grandis (Linn. F) en la zona sur de Costa Rica. Revista Forestal Mesoamericana Kurú, 16(39), 43-52. doi. 10.18845/rfmk.v16i39.4427 